\title{
A comparison of two informative SNP-based strategies for typing Pseudomonas aeruginosa isolates from patients with cystic fibrosis
}

Melanie W Syrmis ${ }^{1,2}$, Timothy J Kidd ${ }^{1,2}$, Ralf J Moser ${ }^{3}$, Kay A Ramsay ${ }^{1,2}$, Kristen M Gibson ${ }^{1}$, Snehal Anuj ${ }^{1}$, Scott C Bell ${ }^{1,4}$, Claire E Wainwright ${ }^{1,5}$, Keith Grimwood ${ }^{1,2}$, Michael Nissen ${ }^{1,2,6}$, Theo P Sloots ${ }^{1,2,6}$ and David M Whiley ${ }^{1,2^{*}}$

\begin{abstract}
Background: Molecular typing is integral for identifying Pseudomonas aeruginosa strains that may be shared between patients with cystic fibrosis (CF). We conducted a side-by-side comparison of two P. aeruginosa genotyping methods utilising informative-single nucleotide polymorphism (SNP) methods; one targeting 10 P. aeruginosa SNPs and using real-time polymerase chain reaction technology (HRM10SNP) and the other targeting 20 SNPs and based on the Sequenom MassARRAY platform (iPLEX20SNP).

Methods: An in-silico analysis of the 20 SNPs used for the iPLEX20SNP method was initially conducted using sequence type (ST) data on the P. aeruginosa PubMLST website. A total of 506 clinical isolates collected from patients attending 11 CF centres throughout Australia were then tested by both the HRM10SNP and IPLEX20SNP assays. Type-ability and discriminatory power of the methods, as well as their ability to identify commonly shared $P$. aeruginosa strains, were compared.

Results: The in-silico analyses showed that the 1401 STs available on the PubMLST website could be divided into 927 different 20-SNP profiles ( $D$-value $=0.999$ ), and that most STs of national or international importance in CF could be distinguished either individually or as belonging to closely related single- or double-locus variant groups. When applied to the 506 clinical isolates, the iPLEX20SNP provided better discrimination over the HRM10SNP method with 147 different 20-SNP and 92 different 10-SNP profiles observed, respectively. For detecting the three most commonly shared Australian P. aeruginosa strains AUST-01, AUST-02 and AUST-06, the two methods were in agreement for 80/81 (98.8\%), 48/49 (97.8\%) and 11/12 (91.7\%) isolates, respectively.

Conclusions: The iPLEX20SNP is a superior new method for broader SNP-based MLST-style investigations of P. aeruginosa. However, because of convenience and availability, the HRM1OSNP method remains better suited for clinical microbiology laboratories that only utilise real-time PCR technology and where the main interest is detection of the most highly-prevalent $P$. aeruginosa CF strains within Australian clinics.
\end{abstract}

Keywords: Pseudomonas aeruginosa, Typing, Cystic fibrosis, MLST, SNP

\footnotetext{
* Correspondence: d.whiley@uq.edu.au

'Queensland Children's Medical Research Institute, The University of Queensland, Brisbane, Queensland 4029, Australia

${ }^{2}$ Queensland Paediatric Infectious Disease Laboratory, Block 28, Royal Children's Hospital, Herston Road, Herston, Brisbane 4029, Queensland, Australia

Full list of author information is available at the end of the article
} 


\section{Background}

Cystic fibrosis (CF) is the most common, lethal autosomal recessive disease in Caucasian populations [1]. Most CF patients die in their third or fourth decade from complications of chronic pulmonary infection. Pseudomonas aeruginosa is the predominant pathogen and once it is established within the lungs of CF patients it is rarely eradicated, resulting in increased treatment requirements and an accelerated decline in lung function, quality of life and survival [2]. While many CF patients acquire $P$. aeruginosa from their natural environment, there is also evidence of person-to-person transmission occurring [3]. Delaying or even preventing $P$. aeruginosa infection is an important management goal. Consequently, determining $P$. aeruginosa acquisition pathways and conducting longitudinal surveillance using molecular-based typing techniques are critical steps for developing novel interventions and evidence-based infection control policies to interrupt the spread of transmissible strains within the CF community [4-6].

Recently, multi-locus sequence typing (MLST) has emerged as an important epidemiological tool for investigating temporally and geographically diverse bacteria [7]. It offers a standardised, reproducible and portable typing approach that allows reliable data comparisons by way of a publically accessible web-based database $[7,8]$. However, when applied to large-scale investigations involving many hundreds or thousands of isolates it is limited by cost and complexity [9]. To circumvent these problems, some researchers have utilised defined sets of informative single nucleotide polymorphisms (SNPs) derived from MLST data to infer genetic relationships between isolates. In essence, it is a narrowed MLST approach and has been applied to various organisms, including pathogens relevant to CF, such as methicillin-resistant Staphylococcus aureus and P. aeruginosa [10-13]. Selection of appropriate SNPs, including SNP location and total numbers, is an integral facet of informative SNP strategy to ensure a discriminatory, yet cost-effective, typing scheme. However, once an informative SNP approach tailored to a particular purpose is implemented, it will theoretically have limitations in terms of discriminatory power if used beyond its original objectives.

Previously, we have shown that SYBR Green-based realtime polymerase chain reaction (PCR) assays and highresolution melting (HRM) curve analysis targeting 10 key SNPs in five housekeeping genes (HRM10SNP) can detect the major $P$. aeruginosa strains shared by CF patients in Queensland, Australia [10]. Furthermore, we demonstrated recently that this form of typing can be adapted to the iPLEX MassARRAY platform to allow highthroughput genotyping [14]. However, based on the high levels of genetic diversity observed amongst shared $P$. aeruginosa strains in the national Australian CF study
[15] and also internationally amongst patients attending CF clinics [16], we sought to reassess the HRM10SNP and investigate alternative SNP-based typing strategies for identifying a broader range of $P$. aeruginosa strains.

\section{Methods \\ Clinical isolates}

To ensure representative and geographical diversity, 506 clinical isolates were sourced randomly from a biobank of CF isolates collected as part of an ongoing national study of shared $P$. aeruginosa strains involving patients attending $11 \mathrm{CF}$ clinics in Australia's five largest cities [15] (Additional file 1: Table S1). Isolates were incubated on horse blood agar plates for 24 -hours at $37^{\circ} \mathrm{C}$. Once purity was confirmed, heat-denatured suspensions of each isolate were prepared as described previously [10].

\section{HRM10SNPAssay}

The HRM10SNP assay was performed for each isolate as described previously [10]. Briefly, each heat-denatured isolate was tested using 10 individual PCR reactions using the qPCR SuperMix-UDG (Invitrogen Australia, Mulgrave, NSW, Australia) on the Rotorgene-6000 (Qiagen, Doncaster, Victoria, Australia). Results from each reaction were compiled to provide a 10-SNP profile for each isolate. As reported previously, isolates with 10SNP profiles of CTCCTCGGCA, TCTTTCGGTA and CCTCCTGATG were determined to be AUST-01, AUST02 and AUST-06, respectively [10].

\section{0-SNP iPLEXMassARRAY(iPLEX20SNP)}

The iPLEX20SNP assay was based on the Sequenom MassARRAY platform (Sequenom, Brisbane, Queensland, Australia) and was a modification of a method described previously [14]. Here, SNPs were derived by analysing sequence data on the $P$. aeruginosa PubMLST website [17]. Briefly, 1070 concatenated sequences of $P$. aeruginosa housekeeping genes (acs $A$, aroE, guaA, mutL, nuoD, ppsA, and $\operatorname{trp} E$ ) were downloaded (12 January, 2012) and investigated for informative SNPs with the aid of the Minimum SNPs software version 2043 [18] and by manual sorting (using BioEdit version 7.0.9.0). Overall, 20 SNPs were identified and SNP positions based on the 2882 bp concatenated $P$. aeruginosa MLST sequence are listed in Tables 1 and 2. Of these 20 SNPs, four were identical to SNPs used in the HRM10SNP assay; SNPs at sites 7, 322, 1152 and 2551 of the iPLEX20SNP assay (Tables 1 and 2) overlapped with SNPs 1, 2, 5 and 10 from the HRM10SNP assay.

Primers and extension primers for each of the 20 SNPs in the iPLEX20SNP were designed as reported previously [14]. All 20 target SNPs were designed for use in a single multiplex well using Assay Designer 4.0 software (Sequenom, Herston, Queensland, Australia). The 


\begin{tabular}{|c|c|c|}
\hline SNP site & $\begin{array}{l}\text { Gene } \\
\text { target }\end{array}$ & Primers $\left(5^{\prime}-3^{\prime}\right)$ \\
\hline \multirow{2}{*}{7 and 45} & \multirow{2}{*}{$\operatorname{acs} A$} & P1 10mer-ACCTTGTGCTTGTCGATGAT \\
\hline & & P2 10mer-GCCACACCTACATCGTCTAT \\
\hline \multirow{2}{*}{322,381 and 387} & \multirow{2}{*}{$\operatorname{acs} A$} & P1 10mer-ATCAGGTTGCCGAGGTTGTC \\
\hline & & P2 10mer-AGACCGGCGCCTGCCTGATG \\
\hline \multirow{2}{*}{416 and 488} & \multirow{2}{*}{$\operatorname{aroE}$} & P1 10mer-TCGGTGTTGTCGCCGCGCAG \\
\hline & & P2 10mer-CAATGTCACCGTGCCGTTCA \\
\hline \multirow{2}{*}{881} & \multirow{2}{*}{$\operatorname{aroE}$} & P1 10mer-CAGAGGAAGAATGCCTCGG \\
\hline & & P2 10mer-CGACATGATGTATGCCAAGG \\
\hline \multirow{2}{*}{894 and 937} & \multirow{2}{*}{ guaA } & P1 10mer-AACATCGTCGACGACGCCAT \\
\hline & & P2 10mer-AACACGCAGGTCAGTTGGTC \\
\hline \multirow{2}{*}{1086 and 1152} & \multirow{2}{*}{ guaA } & P1 10mer-GGCGAGGAACTTCACGTCCTG \\
\hline & & P2 10mer-ATGGGCGTGAAGGTGATCCG \\
\hline \multirow{2}{*}{1297} & \multirow{2}{*}{ mutl } & P1 10mer-AGAAGACCGAGTTCGACCAT \\
\hline & & P2 10mer-AAGATGGTCTTGCCGTTGTG \\
\hline \multirow{2}{*}{1465} & \multirow{2}{*}{ mutl } & P1 10mer-ACCAGCTTGTCGCGCACCAT \\
\hline & & P2 10mer-AGCGCAACGGCCTGCACCT \\
\hline \multirow{2}{*}{1865 and 1958} & \multirow{2}{*}{ nuod } & P1 10mer-TACAGCAGGTGGTTCAGGAT \\
\hline & & P2 10mer-AAGATGGCCGAGCGCCAGT \\
\hline \multirow{2}{*}{2169 and 2208} & \multirow{2}{*}{ ppsA } & P1 10mer-AGAGAAGGGGACCGTCCTG \\
\hline & & P2 10mer-ACCTTGTCCATTTCCGACAC \\
\hline \multirow{2}{*}{2337} & \multirow{2}{*}{ ppsA } & P1 10mer-TGGTCTCCGACATGACCGA \\
\hline & & P2 10mer-TTCGCGAGCGATGATCGCC \\
\hline \multirow{2}{*}{2551} & \multirow{2}{*}{$\operatorname{trp} E$} & P1 10mer-GGATCAACGAAGAGGCCGA \\
\hline & & P2 10mer-TCGATCAGCATCAGGTGCTC \\
\hline
\end{tabular}

$\mathrm{P} 1=$ forward amplification primer;

$\mathrm{P} 2$ = reverse amplification primer;

10 mer $=5^{\prime}$ 10-mer tagACGTTGGATG.

24. amplification primers and 21 extension primers used for SNP detection are listed in Tables 1 and 2. Two extension primers with overlapping mass were used for SNP site 416 to accommodate a known proximal SNP variation (Table 2).

SNP detection by MassARRAY was performed as outlined formerly [14], with the following modifications: (1) following the initial PCR, residual PCR Taq polymerase was removed by protease digestion; $1 \mu \mathrm{l}$ of protease solution (1.07 AU, Qiagen, Doncaster, Victoria, Australia) was added to each PCR reaction and the mixture incubated at $55^{\circ} \mathrm{C}$ for $30 \mathrm{~min}$ followed by an inactivation at $95^{\circ} \mathrm{C}$ for $5 \mathrm{~min}$; and (2) the single base extension step was performed using the iPLEX Pro Extension Reaction Kit (Sequenom, Herston, Queensland, Australia) following manufacturer's instructions. SNPs were coded from 1 to 20 to generate a 20 SNP code. The 20-SNP profiles were then interpreted using the data compiled from in-silico analysis of the $P$. aeruginosa MLST database, as described below, to provide predicted sequence types (STs). Characterised isolates representative of each SNP were used as reference controls for each test run.

\section{In-silico analysis of 20-SNP profiles from the MLST database}

For final result analyses, 1779 concatenated sequences of $P$. aeruginosa housekeeping genes were again downloaded (13 ${ }^{\text {th }}$ December 2012) and reanalysed. The 1779 $P$. aeruginosa sequences yielded 1401 different STs. The predicted ability of the 20-SNP profile to distinguish these 1401 STs was investigated, as was its ability to distinguish STs of national: AUST-01 (ST- 649), AUST-02 (ST-775), AUST-03 (ST-242), AUST-04 (ST-788), AUST05 (STs 274 and 781), AUST-06 (ST-801), AUST-07 (ST262), AUST-08 (STs 782, 783, 784 and 785) and AUST-09 (STs 274 and 1043), AUST-10 (STs 155 and 179), AUST11 (STs 803, 1034, 1037, 508, 804, 822 and 882), AUST-12 (ST-179), AUST-13 (STs 389 and 800), AUST-14 (STs 155 and 179), AUST-15 (ST-17), AUST-16 (ST-905), AUST-17 (ST-810), AUST-18 (ST-274), AUST-19 (STs 155 and 786), AUST-20 (ST-655), AUST-21 (STs 808), AUST-22 (ST-809), AUST-23 (ST-833), AUST-24 (ST-308), AUST25 (ST-274), AUST-26 (ST-179), AUST-27 (ST-455), AUST-28 (ST-241), AUST-29 (ST-261), AUST-30 (ST1036), AUST-31 (ST-274), AUST-32 (ST-236), AUST-33 (ST-12), AUST-34 (ST-1038), AUST-35 (ST-553), AUST36 (ST-277), AUST-37 (ST-155), AUST-38 (STs 254 and 1041) [15], and international importance: LES (ST-146), Manchester (ST-217), DK2 (ST-386), PA01 (ST-549), PA14 (ST-253), M18 (ST-1239), PACS2 (ST-1394), NCGM2.S1 (ST-235), PA7 (ST-1195), Clone C (ST-17), Dutch-1 (ST406), Dutch-2 (ST-497) and Midlands (ST-148) $[17,19,20]$.

\section{Statistical analysis}

Discriminatory power and the quantitative measure of congruence between the HRM10SNP and iPLEX20SNP methods and corresponding 95\% confidence intervals (CI) were determined by calculating the Simpson's Index of Diversity and the adjusted Wallace coefficients respectively using the online analysis tool at http://darwin.phyloviz.net/ ComparingPartitions/index.php?link=Tool. The 20-SNP profile in-silico data were used to predict STs for the 506 clinical isolates utilising the experimental results from the iPLEX20SNP assay.

\section{Results}

\section{In-silico analysis of MLST data}

Analysis of the $P$. aeruginosa PubMLST website [17] $\left(13^{\text {th }}\right.$ December 2012) showed that the 1401 STs could be divided into 927 different 20-SNP profiles (Additional file 2: Table S2). Overall, 711 STs could be distinguished individually by the 20-SNP profile, whereas the remaining 690 STs had overlapping 20-SNP profiles with one $(n=120)$, 
Table 2 Extension primers used for the iPLEX20SNP

\begin{tabular}{|c|c|c|c|c|c|c|c|}
\hline SNP site ${ }^{a}$ & Gene target & UEP $\left(5^{\prime}-3^{\prime}\right)$ & Mass & EP1, mass & EP2, mass & EP3, mass & EP4, mass \\
\hline 7 & $\operatorname{acs} A$ & ACATCGTCTATGGCCCG & 5146.4 & C,5393.5 & $\mathrm{T}, 5473.5$ & & \\
\hline 45 & $\operatorname{acs} A$ & ggggTCTGTTCGAGGGCGT & 5931.8 & $\mathrm{~A}, 6203$ & $\mathrm{G}, 6219$ & & \\
\hline 322 & $\operatorname{acs} A$ & gggtaGCGTGGGCGCCCGGCA & 6529.2 & $\mathrm{~T}, 6800.4$ & C,6816.4 & & \\
\hline 381 & ascA & ttcccAGCCGTTCTTCGGCGTGGT & 7302.7 & C,7549.9 & A,7573.9 & G,7589.9 & $\mathrm{T}, 7629.8$ \\
\hline 387 & $\operatorname{asc} A$ & gaaGAGGTTGTCCACCAG & 5548.6 & G,5795.8 & A,5875.7 & & \\
\hline \multirow{2}{*}{$416^{b}$} & \multirow{2}{*}{$\operatorname{aroE}$} & TGCCGTTCAAGGAAGA & \multirow{2}{*}{4930.2} & \multirow{2}{*}{ C,5177.4 } & \multirow{2}{*}{ A,5201.4 } & \multirow{2}{*}{ G,5217.4 } & \\
\hline & & aGgCGTTCAAGGAACT & & & & & \\
\hline 488 & $\operatorname{aro} E$ & cCCACCCTGATCCGCCT & 5027.3 & C,5274.5 & G,5314.5 & $\mathrm{T}, 5354.4$ & \\
\hline 881 & aroE & gagggTGTATGCCAAGGAACCGAC & 7451.9 & C,7699 & G,7739.1 & T,7778.9 & \\
\hline 894 & guaA & gTCCTCCAAGGTCCTGCT & 5426.5 & C,5673.7 & A,5697.7 & G,5713.7 & \\
\hline 937 & guaA & tgtTCcgCGATGGCCTTGTGCA & 6733.4 & $\mathrm{~T}, 7004.6$ & C,7020.6 & & \\
\hline 1086 & guaA & gtCGAGGACAAGTTCCTCGG & 6158 & C,6405.2 & G,6445.2 & $\mathrm{T}, 6485.1$ & \\
\hline 1152 & guaA & ctcaGCACCTTCATCGAAGT & 6036.9 & C,6284.1 & G,6324.2 & $\mathrm{T}, 6364$ & \\
\hline 1297 & mutl & GTGGAAAGCCACGTCGAA & 5557.6 & $\mathrm{~T}, 5828.8$ & C,5844.8 & & \\
\hline 1465 & mutL & gggCGGCtcGCACCTGTGGGG & 6520.2 & $C, 6767.4$ & $\mathrm{~T}, 6847.3$ & & \\
\hline 1865 & nuoD & agagaCAGTCtcGGCACAGTTCAT & 7666 & C,7913.2 & Т,7993.1 & & \\
\hline 1958 & nuoD & $\operatorname{tgctGATCACGTCGACCCGCTG~}$ & 6687.3 & $\mathrm{G}, 6934.5$ & C,6974.5 & & \\
\hline 2169 & ppsA & ccggaGCGCTGGCCGATGGCACG & 7091.6 & $\mathrm{G}, 7338.8$ & $\mathrm{~T}, 7362.8$ & C,7378.8 & A,7418.7 \\
\hline 2208 & ppsA & CCGACACGTCGTTGATCAC & 5748.7 & G,5995.9 & A,6075.8 & & \\
\hline 2337 & ppsA & CGCCGCGTGGCAGGT & 4610 & $\mathrm{~T}, 4881.2$ & C,4897.2 & & \\
\hline 2551 & $\operatorname{trpE}$ & CAGATCCTGCTCCAG & 4512.9 & $\mathrm{G}, 4760.1$ & $C, 4800.2$ & $\mathrm{~A}, 4840$ & \\
\hline
\end{tabular}

Non-template bases are indicated in lower case. Mass (Daltons) is provided for the unextended extension primer (UEP), as well as associated extension products (EP) 1,2 and where relevant 3 and 4.

${ }^{\text {a }} \mathrm{SNP}$ position is based on the $2882 \mathrm{bp}$ concatenated sequence.

${ }^{b}$ Note that SNP 416 had two extension primers to accommodate a known proximal SNP variation.

two $(n=51)$, three $(n=13)$, four $(n=11)$, five $(n=6)$, six $(n=2)$, seven $(n=2)$, eight $(n=1)$, nine $(n=2)$, ten $(n=4)$, $11(\mathrm{n}=2), 12(\mathrm{n}=1)$, or 13 other $(\mathrm{n}=1)$ STs (Additional file 2: Table S2). In total, 486/690 (70.4\%) STs showing overlapping 20-SNP profiles comprised closely related single- or double-locus variant STs. Based on these data, the $D$-value for the 20-SNP profiling method was calculated as 0.999 (95\% CI 0.998, 0.999). For the STs of national and international importance, nine could be distinguished individually by the 20-SNP profile, whereas the remaining exhibited overlapping 20-SNP profiles. Most of the latter were again single- or double-locus variant STs (Table 3 and Additional file 2: Table S2).

\section{HRM10SNP and iPLEX20SNP typing of the 506 clinical isolates}

Application of the HRM10SNP assay provided complete 10-SNP profiles for 494/506 isolates (type-ability $=97.6 \%$ ) of which 92 different 10-SNP profiles were observed; 12 isolates were not typed using the HRM10SNP method as one or more SNPs failed to be called by the HRM analysis (Additional file 3: Table S3). The iPLEX20SNP assay provided complete 20 -SNP profiles for 471/506 isolates (type- ability $=93.1 \%$ ) of which there were 147 distinct 20-SNP profiles; 35 isolates failed to provide complete 20-SNP profiles due to the iPLEX20SNP assay failing to characterise one or more SNPs (Additional file 3: Table S3). When the 147 complete 20 -SNP profiles (471 isolates) from the iPLEX20SNP assay were used to predict a MLST type (based on the data provided in Additional file 2: Table S2), 124 of $147(84.4 \%)$ profiles matched profiles obtained from the MLST website and there could provide a predicted MLST type or types. Twenty-three 20-SNP profiles from 28 isolates did not match with any of the listed 20-SNP profiles in Additional file 2: Table S2, and therefore a MLST type could not be predicted.

Overall, 470 isolates provided complete SNP profiles by both the HRM10SNP and iPLEX20SNP assays. Simpson's Index of Diversity and adjusted Wallace coefficients between the HRM10SNP and iPLEX20SNP methods were calculated using these 470 isolates (Table 4). Simpson's Index of Diversity of the iPLEX20SNP (0.947) was similar to that of the HRM10SNP method (0.944). However, when concordance between the assays was assessed using the adjusted Wallace coefficient, the iPLEX20SNP method (94.9\%) was a better predictor of the HRM10SNP method 
Table $3 P$. aeruginosa MLST data from the $P$. aeruginosa MLST database website $\left(13^{\text {th }}\right.$ December 2012$)$ and associated SNP profiles for STs of national and international importance

\begin{tabular}{|c|c|c|c|}
\hline 20 SNP profile ${ }^{a}$ & MLST $^{\mathbf{b}}$ & $\begin{array}{l}\text { Total } \\
\text { STs }\end{array}$ & $\begin{array}{l}\text { Related STs } \\
\text { (SLV or DLV') }\end{array}$ \\
\hline CGTCGACTACCTCCCCGGTA & 649(AUST-01) & 1 & \\
\hline TGCAAGCTACCTTCCCGGTA & 775(AUST-02) & 1 & \\
\hline TGCAAGCTCCCCTTTGGGCG & 242(AUST-03), 996 & 2 & $2 / 2=S L V s$ \\
\hline TGCCGGCTATCCCCCCGACA & 787, 788(AUST-04) & 2 & $2 / 2=S L V s$ \\
\hline TGTCGGCTACCTITCGGGTA & $\begin{array}{c}\text { 209, 268, 274(AUST-05,-09, -18,-25, \&-31) , 466, 546, 781(AUST-05), } \\
\text { 936, 1043(AUST-09), 1068, 1089, 1301, } 1326\end{array}$ & 12 & $12 / 12=S L V s$ \\
\hline CGCAAGCTATCCCCCGGGTG & 4, 801 (AUST-06), 1292 & 3 & $2 / 3=S L V s$ \\
\hline CGCAAGCTATCCCCCCGGCA & 262(AUST-07), 774, 1165 & 3 & $3 / 3=S L V s$ \\
\hline CGCAGGGCCCCCTTCGGGCG & 782(AUST-08), 783(AUST-08), 784(AUST-08), 785(AUST-08) & 4 & $4 / 4=S L V S$ \\
\hline CGCCGGCTCCCCCCCCGACA & 179(AUST-10,-12,-14,\&-26), 180, 353 & 3 & $3 / 3=S L V s$ \\
\hline TGCAAGCTACCCCCTGGACA & 13, 155(AUST-10,-14, -19, \&-37), 280, 541, 579, 677, 786(AUST-19), 1276, 1316, 1335 & 10 & $10 / 10=S L V s$ \\
\hline CGCAAGCTACCTCCCCGGTA & 384, 1037(AUST-11) & 2 & \\
\hline CGCAGGCTACCTCCTGGGTG & 508(AUST-11), 937 & 2 & $2 / 2=S L V s$ \\
\hline TGCCGGCTCCCCCCTGGGCA & $554, \mathbf{8 0 4 ( A U S T - 1 1 )}$ & 2 & $2 / 2=S L V s$ \\
\hline CGCAGGCTACCTCCCCGGTA & 589, 791, 803(AUST-11) & 3 & $3 / 3=S L V s$ \\
\hline TGCCGGCTATCCCCCCGGCA & 822(AUST-11), 1239(M18) & 2 & $2 / 2=S L V s$ \\
\hline CGCAAGCTACCTCCCCAGTA & 882(AUST-11), 1151, 1233 & 3 & $3 / 3=S L V S$ \\
\hline CGCAGGCTACCTTCCCGGTA & 1034(AUST-11) & 1 & \\
\hline CGCAAGGTACCTCCTGGGCG & 800(AUST-13) & 1 & \\
\hline CGCAAGGTCCCCCCCGGGTG & 389(AUST-13) & 1 & \\
\hline CGTCGGCTATCCTTCCGGTA & 17(AUST-15 \& Clone C), 318, 322, 380, 636, 688, 845, 958, 1255, 1313 & 10 & $9 / 10=S L V s$ \\
\hline CGCAAGCTACTCTCCCGGTG & 905(AUST-16), 1039 & 2 & \\
\hline CGTCGGCTATCCCCTGGGCA & 398, 399, 401, 810(AUST-17) & 4 & $3 / 4=S L V s$ \\
\hline CGTCGGCCACTCTTCCGGCG & 655(AUST-20), 709 & 2 & $2 / 2=S L V s$ \\
\hline TGCAAGCTACCCTCCCGGTA & 669, 808(AUST-21) & 2 & $2 / 2=S L V s$ \\
\hline CGCCGGGCCTCCTCTGAGTG & 809(AUST-22) & 1 & \\
\hline TGTCGGCTACCTTCCCGGTA & 833(AUST-23), 839 & 2 & $2 / 2=S L V s$ \\
\hline TACCAGGCCCCCTCCGAGTG & 89, 307, 308(AUST-24), 662, 1028 & 5 & $2 / 5=\mathrm{DLVs}$ \\
\hline TGTCGGCCCCCCTTCGGGTA & 455(AUST-27) & 1 & \\
\hline CGCAAGCTACCTTCCCGGTA & 232, 241(AUST-28), 247, 379, 471, 577 & 6 & $5 / 6=S L V S$ \\
\hline CGTCGGCTATCCCCTGGGTA & 169, 261(AUST-29) & 2 & \\
\hline CGCAGACTCCCCTCCCGGTA & 1036(AUST-30) & 1 & \\
\hline TGCAAGCTATCCTCCCAGCG & 236(AUST-32), 239, 240 & 3 & $3 / 3=S L V S$ \\
\hline TGCAAGCTATCCCCCCGGTG & 12(AUST-33) & 1 & \\
\hline CGCAAGCTCCCCCCCGGGTA & 103, 244, 441, 462, 464, 594, 766, 986, 1038(AUST-34), 1181, 1227, 1338 & 12 & $10 / 12=S L V s$ or DLVs \\
\hline CGCAAGCTATCCTTCCGGTA & 445, 553(AUST-35) & 2 & \\
\hline CGCAAGCTCCTCTITCGGTA & 277(AUST-36), 364, 1128, 1390 & 4 & $4 / 4=S L V s$ \\
\hline TGTCGGCTCCTCTITGGGTA & 254(AUST-38), 1041(AUST-38) & 2 & $2 / 2=S L V S$ \\
\hline TGTCGGCTACCTCCCCGGTG & 146(LES), 374, 467, 681, 683, 970 & 6 & $6 / 6=S L V S$ \\
\hline TGCAAGCTACCTCCCCGACG & 217(Manchester), 1134 & 2 & $2 / 2=S L V S$ \\
\hline CGCAAGCTACCTCCCCGGCG & 386(DK2), 1244 & 2 & \\
\hline TGCAGGCTCCCCCCCCAGCA & 549(PA01), 1331 & 2 & $2 / 2=S L V s$ \\
\hline TATCGGGCCCCCTCCGAGTG & 65,107, 109, 253(PA14), 297, 338, 342, 377, 532, 773, 815, 923, 1110, 1363 & 14 & $3 / 14=S L V s$ or DLVs \\
\hline
\end{tabular}


Table $3 P$. aeruginosa MLST data from the $P$. aeruginosa MLST database website $\left(13^{\text {th }}\right.$ December 2012$)$ and associated SNP profiles for STs of national and international importance (Continued)

\begin{tabular}{|c|c|c|c|}
\hline CGTCGGCTCTCCCCTGGACA & 1394(PACS2) & 1 & \\
\hline CGCTAGGCCCCCTCCGAGTG & 227, 230, 235(NCGM2.S1), 533, 534, 696, 745, 976, 989 & 9 & $9 / 9=S L V S$ \\
\hline CGCGGAGCCCTCTCCGTGTG & 366, 368, 1006, 1063, 1190, 1191, 1195(PA7) & 7 & $4 / 7=S L V S$ \\
\hline CGTCGGCTACCTCCCCGACA & 406(Dutch-1), 484, 519, 536, 547, 575, 608, 1214, 1235, 1312, 1318 & 11 & $10 / 11=$ SLVS or DLVS \\
\hline CGTCGGCTATCCTTGGGTA & 497(Dutch-2), 544, 895, 1317 & 4 & $4 / 4=S L V s$ or DLVs \\
\hline CGCAAGCTATCCCCCGAGTA & 138, 140, 148(Midlands), 956 & 4 & $2 / 4=S L V S$ \\
\hline
\end{tabular}

The STs consistent with recognised $P$. aeruginosa strains are indicated in parentheses.

${ }^{a}$ SNP profile is in the order of $7,45,322,381,387,416,488,881,894,937,1086,1152,1297,1465,1865,1958,2169,2208,2337$ and 2551. These SNPs were derived from the sequence data from the Pseudomonas aeruginosa MLST database website (http://darwin.phyloviz.net/ComparingPartitions/index.php?link=Tool) on 13 December 2012. ${ }^{b}$ MLST types available from the Pseudomonas aeruginosa MLST database website (http://pubmlst.org/paeruginosa) on 13 December 2012.

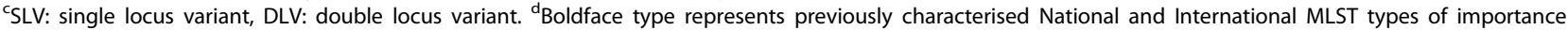
(e.g., AUST-01 has the MLST type 649).

than vice versa $(89 \%)$. To investigate the latter further we identified all 10-SNP profiles that were further discriminated by the 20-SNP profiles (Additional file 4: Table S4); 34 HRM10SNP profiles were further distinguished into 10120 -SNP profiles using the iPLEX20SNP method. Of note, these involved $30 \mathrm{STs}$ associated with CF strains of local or international importance (Additional file 4: Table S4). In contrast, there were only 11 iPLEX20SNP profiles that were further discriminated by the HRM10SNPassay (Additional file 3: Table S3).

Given the high prevalence of AUST-01, AUST-02 and AUST-06 in Australia, and that the HRM10SNP assay was primarily designed to target these strains, we compared the ability of both assays to distinguish these strains. For isolates identified as AUST-01, AUST-02 or AUST-06 by either method (Additional file 3: Table S3), the results of the two methods were in agreement for 80/81 (98.8\%), 48/49 (97.8\%) and 11/12 (91.7\%) isolates, respectively. Both isolates giving discrepant results for AUST-01 and AUST-06 were identified as AUST-01 or AUST-06 by the iPLEX20SNP method, but not by the HRM10SNP assay. For both of these isolates, their 10SNP profiles by the HRM10SNP differed by only one SNP from the expected profiles of AUST-01 and AUST-06. Upon repeat testing in the HRM10SNP assay, both subsequently typed as AUST-01 and AUST-06, suggesting that

Table 4 Number of types, Simpson's index of diversity and adjusted Wallace coefficients for the HRM10SNP and iPLEX20SNP assays calculated from application to the $\mathbf{4 7 0}$ isolates providing complete SNP profiles by both methods

\begin{tabular}{lccc}
\hline Assay & No. of types & $\begin{array}{c}\text { Simpson's index } \\
\text { of diversity } \\
\mathbf{( 9 5 \% ~ C l )}\end{array}$ & $\begin{array}{c}\text { Adjusted Wallace } \\
\text { coefficient } \\
\mathbf{( 9 5 \% ~ C l )}\end{array}$ \\
\hline HRM10SNP & 91 & 0.944 & 0.897 \\
20SNP iPLEX & 147 & $(0.933-0.955)$ & $(0.876-0.918)$ \\
& & 0.947 & 0.949 \\
& & $(0.936-0.959)$ & $(0.912-0.986)$ \\
\hline
\end{tabular}

there was a mistake in the original HRM10SNP testing. The discordant result for AUST-02 was associated with a different ST; one isolate was identified as AUST-02 by the HRM10SNP method, but differed by two SNPs from the expected 20-SNP profile for AUST-02 in the iPLEX20SNP assay (predicted MLST type of 778).

\section{Discussion}

The in-silico analyses of sequence data from the P. aeruginosa PubMLST website showed that more than half of recognised STs could be distinguished individually by the 20-SNP profile of the iPLEX20SNP assay. Furthermore, the recognised STs that were unable to be distinguished by this assay were typically single- or double-locus variants. Hence, theoretically the iPLEX20SNP method has considerable potential for broader-based MLST-focused studies of $P$. aeruginosa, here and elsewhere. As the iPLEX20SNP is also based on the Sequenom MassARRAY platform, it is particularly suitable for high-throughput investigations [14]. Using this technology up to 384 isolates can be tested within one working day for less than \$AUD 10 per isolate [14], and is therefore quite favourable compared to other technologies. For example, for our 506 test isolates we estimate that classical DNA sequencingbased MLST would have cost approximately \$AUS 60,720 (\$AUS 120 per isolate), whereas costs for the HRM10SNP and iPLEX20SNP methods were approximately \$AUS 10,120 (\$AUS 20 per isolate) ${ }^{13}$ and \$AUS 5,060 respectively.

Compared to the HRM10SNP, the iPLEX20SNP method clearly provided better discrimination when applied to the $P$. aeruginosa test isolates used in this study. Of note was that the HRM10SNP assay grouped numerous unrelated isolates, including STs of shared strains in the CF patient population, while the iPLEX20SNP method was able to distinguish between these isolates (Additional file 4: Table S4). This was likely due to the higher number of SNPs and that SNP selection for iPLEX20SNP was based on a large international MLST database. These observations provide experimental data to support the above 
in-silico analyses. Indeed, in the clinical context, attaining optimal discriminatory power is particularly important when trying to identify new or emerging shared $P$. aeruginosa strains in CF patients. Consequently, iPLEX20SNP is ideally suited for broader, investigatory studies of $P$. aeruginosa infected patients.

While the HRM10SNP lacked overall discriminatory power, it nevertheless proved to be well-suited for detecting AUST-01, AUST-02 and AUST-06 amongst $P$. aeruginosa isolates from a broad range of Australian CF clinics. AUST-01 and AUST-02 are the shared P. aeruginosa strains of greatest concern in Australia [15], and therefore simple methods for detecting these strains remain of local clinical and research interest. The one key benefit of the HRM10SNP method is that it is based on real-time PCR technology, which is now commonplace in most clinical microbiology laboratories. Hence, the HRM10SNP method may still be a useful diagnostic tool locally for laboratories with no access to specialised equipment such as the Sequenom MassARRAY platform.

Limitations in terms of typeability (i.e., the number of isolates providing complete SNP profiles) were observed, however, with $2.4 \%$ and $6.9 \%$ of isolates failing to give complete profiles in the HRM10SNP and the iPLEX20SNP assays respectively. Typically these problems are caused by poor isolate preparation (i.e., insufficient DNA) or otherwise sequence variation in primer targets $[10,14]$. Given the sheer diversity amongst the $P$. aeruginosa MLST housekeeping genes, it is highly likely that sequence variation would account for a large proportion of the problems observed here. In any event, we do not see this as an important limitation affecting the broader utility of the assays given that other methods, such as DNA sequencing, could be applied if necessary to the small numbers of untypeable isolates.

\section{Conclusions}

In summary, molecular typing is an integral part of investigating the development and spread of shared $P$. aeruginosa strain genotypes in patients with CF. The iPLEX20SNP is a superior new method providing sufficient throughput and discriminatory power for broader SNP-based MLSTstyle investigations of $P$. aeruginosa, whereas the HRM10SNP method remains a convenient technique for screening CF clinical isolates for the current most commonly shared Australian P. aeruginosa strains and should be able to be performed by most clinical microbiology laboratories.

\section{Availability of supporting data}

The data sets supporting the results of this article are included within the article and its additional files.

\section{Additional files}

Additional file 1: Table S1. Source and distribution of Pseudomonas aeruginosa isolates from cystic fibrosis patients.

Additional file 2: Table S2. $P$. aeruginosa MLST data from the $P$. aeruginosa MLST database website $\left(13^{\text {th }}\right.$ December 2012) and associated SNP profiles. iPLEX20SNP predicted STs consistent with recognised $P$. aeruginosa strains are indicated in parentheses.

Additional file 3: Table S3. IPLEX20SNP and HRM10SNP results for the 506 isolates. iPLEX20SNP predicted STs or HRM10SNP profiles consistent with recognised $P$. aeruginosa strains are indicated in parentheses.

Additional file 4: Table S4. HRM10SNP profiles further discriminated by the iPLEX20SNP assay. iPLEX20SNP predicted STs or HRM10SNP profiles consistent with recognised $P$. aeruginosa strains are indicated in parentheses.

\section{Abbreviations}

Cl: Confidence interval; CF: Cystic fibrosis; HRM: High-resolution melting; MLST: Multi-locus sequence typing; PCR: Polymerase chain reaction; STs: Sequence types; SNPs: Single nucleotide polymorphisms.

\section{Competing interests}

The authors have no competing interests to declare.

\section{Authors' contributions}

Conceptualisation by MS, TK, SB, CW, KG, MN, TS, and DW; development by MS, TK, SA, and DW; analysis and interpretation by MS, TK, KR, SA, KG, KMG, DW; the initial drafts were written by MS, TK, KG and DW. All authors provided feedback and have read and approved the final version of the manuscript.

\section{Authors' information}

Melanie W Syrmis and Timothy J Kidd equal first authors.

\section{Acknowledgements}

We thank the ACPinCF Investigator Group and the participants, research and clinical staff, and clinical microbiology laboratories at each of the study sites for their assistance with sample collection (See reference 15 for full details). We gratefully acknowledge Dr Robert Ware of the Queensland Children's Medical Research Institute and School of Population Health, The University of Queensland for his assistance with sample randomisation and selection. This publication made use of the Pseudomonas aeruginosa MLST website (http://darwin.phyloviz.net/ComparingPartitions/index.php?link=Tool) developed by Keith Jolley and sited at the University of Oxford (Jolley \& Maiden, BMC Bioinformatics 2010, 11:595). The development of this site has been funded by the Wellcome Trust.

\section{Funding}

This study was supported by the Australian Cystic Fibrosis Research Trust and The Children's Health Foundation Queensland. The National Health and Medical Research Council Project Grant 455919, The Children's Health Foundation Queensland, The Queensland Health Office of Health and Medical Research, The Australian Cystic Fibrosis Research Trust, The Prince Charles Hospital Foundation and Rotary Australia supported the collection of the isolates used in this project.

\section{Author details}

${ }^{1}$ Queensland Children's Medical Research Institute, The University of Queensland, Brisbane, Queensland 4029, Australia. ${ }^{2}$ Queensland Paediatric Infectious Disease Laboratory, Block 28, Royal Children's Hospital, Herston Road, Herston, Brisbane 4029, Queensland, Australia. ${ }^{3}$ Sequenom Inc., Sequenom Asia Pacific, Brisbane, Queensland 4029, Australia. ${ }^{4}$ Department of Thoracic Medicine, The Prince Charles Hospital, Brisbane, Queensland 4032, Australia. ${ }^{5}$ Queensland Children's Respiratory Centre, Royal Children's Hospital, Brisbane, Queensland 4029, Australia. ${ }^{6}$ Microbiology Division, Pathology Queensland Central Laboratory, Brisbane, Queensland 4029, Australia.

Received: 28 October 2013 Accepted: 28 May 2014

Published: 5 June 2014 


\section{References}

1. O'Sullivan BP, Freedman SD: Cystic fibrosis. Lancet 2009, 373:1891-1904

2. Hauser AR, Jain M, Bar-Meir M, McColley SA: Clinical significance of microbial infection and adaption in cystic fibrosis. Clin Microbiol Rev 2011, 24:29-70.

3. Fothergill JL, Walshaw MJ, Winstanley C: Transmissible strains of Pseudomonas aeruginosa in cystic fibrosis lung infections. Eur Respir J 2012, 40:227-238.

4. Ashish A, Shaw M, Winstanley C, Humphrey L, Walshaw MJ: Halting the spread of epidemic Pseudomonas aeruginosa in an adult cystic fibrosis centre: a prospective cohort study. J R Soc Med Short Rep 2013, 4:1.

5. Kidd T, Grimwood K, Ramsay K, Rainey P, Bell S: Comparison of three molecular techniques for typing Pseudomonas aeruginosa isolates in sputum samples from patients with cystic fibrosis. J Clin Microbiol 2011, 49:263-268.

6. Sullivan CB, Diggle MA, Clarke SC: Multilocus sequence typing: data analysis in clinical microbiology and public health. Mol Biotech 2005, 29:245-254.

7. Maiden M: Multilocus sequence typing of bacteria. Ann Rev Microbiol 2006, 60:561-588

8. Pérez-Losada M, Cabezas P, Castro-Nallar E, Crandall KA: Pathogen typing in the genomics era: MLST and the future of molecular epidemiology. Infect Gen Evol 2013, 16:38-53.

9. Li W, Raoult D, Fournier PE: Bacterial strain typing in the genomic era. FEMS Microbiol Rev 2009, 33:892-916.

10. Anuj SN, Whiley DM, Kidd TJ, Ramsay KA, Bell SC, Syrmis MW, Grimwood K, Wainwright CE, Nissen MD, Sloots TP: Rapid single-nucleotide polymorphism-based identification of clonal Pseudomonas aeruginosa isolates from patients with cystic fibrosis by the use of real-time PCR and high-resolution melting curve analysis. Clin Microbiol Infect 2011, 17:1403-1408.

11. Huygens F, Inman-Bamber J, Nimmo GR, Munckhof W, Schooneveldt J, Harrison B, McMahon JA, Giffard PM: Staphylococcus aureus genotyping using novel real-time PCR formats. J Clin Microbiol 2006, 44:3712-3719.

12. Robertson GA, Thiruvenkataswamy $V$, Shilling $H$, Price $E P$, Huygens $F$, Henskens FA, Giffard PM: Identification and interrogation of highly informative single nucleotide polymorphism sets defined by bacterial multilocus sequence typing databases. J Med Microbiol 2004 53:35-45.

13. Eusebio N, Pinheiro T, Amorim AA, Gamboa F, Saraiva L, Gusmão L, Amorim A, Araujo R: SNaPaer: a practical single nucleotide polymorphism multiplex assay for genotyping of Pseudomonas aeruginosa. PLoS One 2013, 8:e66083.

14. Syrmis MW, Moser RJ, Kidd TJ, Hunt P, Ramsay KA, Bell SC, Wainwright $C E$, Grimwood K, Nissen MD, Sloots TP, Whiley D: High-throughput single nucleotide polymorphism-based typing of shared Pseudomonas aeruginosa strains in cystic fibrosis patients using the Sequenom iPLEX platform. J Med Microbiol 2013, 62:734-740.

15. Kidd TJ, Ramsay KA, Hu H, Marks GB, Wainwright CE, Bye PT, Elkins MR Robinson PJ, Rose BR, Wilson JW, Grimwood K, Bell SC: Shared Pseudomonas aeruginosa genotypes are common in Australian cystic fibrosis centres. Eur Respir J 2013, 41:1091-1100.

16. Logan C, Habington A, Lennon G, Grogan J, Byrne M, O'Leary J, O'Sullivan $\mathrm{N}$ : Genetic Relatedness of Pseudomonas aeruginosa isolates among a paediatric cystic fibrosis patient cohort in Ireland. J Med Microbiol 2012, 61:64-70.

17. Pseudomonas aeruginosa MLST Database Home Page. [http://pubmlst. org/paeruginosa/

18. Price EP, Inman-Bamber J, Thiruvenkataswamy V, Huygens F, Giffard PM: Computer-aided identification of polymorphism sets diagnostic for groups of bacterial and viral genetic variants. BMC Bioinform 2007, $8: 278$

19. Winsor GL, Lam DK, Fleming L, Lo R, Whiteside MD, Yu NY, Hancock RE, Brinkman FS: Pseudomonas Genome Database: improved comparative analysis and population genomics capability for Pseudomonas genomes. Nucleic Acids Res 2011, 39:D596-D600.
20. De Soyza A, Hall AJ, Mahenthiralingam E, Drevinek P, Kaca W, Drulis-Kawa Z, Stoitsova SR, Toth V, Coeyne T, Zlosnik JEA, Burns JL, Sá-Correia I, De Vos D, Pirnay JP, Kidd T, Reid D, Manos J, Klockgether J, Wiehlmann L, Tümmler B, McClean S, Winstanley C, on behalf of EU FP7 funded COST Action BM1003 "Cell surface virulence determinants of Cystic Fibrosis pathogens: Developing an international Pseudomonas aeruginosa reference panel. Microbiologyopen 2013, 2:1010-1023.

doi:10.1186/1471-2334-14-307

Cite this article as: Syrmis et al: A comparison of two informative SNP-based strategies for typing Pseudomonas aeruginosa isolates from patients with cystic fibrosis. BMC Infectious Diseases 2014 14:307.

\section{Submit your next manuscript to BioMed Central and take full advantage of:}

- Convenient online submission

- Thorough peer review

- No space constraints or color figure charges

- Immediate publication on acceptance

- Inclusion in PubMed, CAS, Scopus and Google Scholar

- Research which is freely available for redistribution

Submit your manuscript at www.biomedcentral.com/submit
Biomed Central 